Note

\title{
CHARACTERIZATION OF LINE-CONSISTENT SIGNED GRAPHS
}

\author{
Daniel C. Slilaty \\ Wright State University \\ Dayton, $\mathrm{OH}$ 45435-0001, USA \\ e-mail: daniel.slilaty@wright.edu \\ AND \\ Thomas ZasLaVsky \\ Binghamton University (SUNY) \\ Binghamton, NY 13902-6000, USA \\ e-mail: zaslav@math.binghamton.edu
}

\begin{abstract}
The line graph of a graph with signed edges carries vertex signs. A vertex-signed graph is consistent if every circle (cycle, circuit) has positive vertex-sign product. Acharya, Acharya, and Sinha recently characterized line-consistent signed graphs, i.e., edge-signed graphs whose line graphs, with the naturally induced vertex signature, are consistent. Their proof applies Hoede's relatively difficult characterization of consistent vertex-signed graphs. We give a simple proof that does not depend on Hoede's theorem as well as a structural description of line-consistent signed graphs.
\end{abstract}

Keywords: line-consistent signed graph, line graph, consistent vertex-signed graph, consistent marked graph.

2010 Mathematics Subject Classification: 05C22, 05C76.

\section{REFERENCES}

[1] B.D. Acharya, A characterization of consistent marked graphs, Nat. Acad. Sci. Letters (India) 6 (1983) 431-440.

[2] B.D. Acharya, Some further properties of consistent marked graphs, Indian J. Pure Appl. Math. 15 (1984) 837-842.

[3] B.D. Acharya, M. Acharya and D. Sinha, Cycle-compatible signed line graphs, Indian J. Math. 50 (2008) 407-414. 
[4] B.D. Acharya, M. Acharya and D. Sinha, Characterization of a signed graph whose signed line graph is S-consistent, Bull. Malaysian Math. Sci. Soc. (2) 32 (2009) $335-341$.

[5] M. Acharya, ×-line signed graphs, J. Combin. Math. Combin. Comput. 69 (2009) $103-111$.

[6] M. Behzad and G. Chartrand, Line-coloring of signed graphs, Elem. Math. 24 (1969) $49-52$.

[7] L.W. Beineke and F. Harary, Consistent graphs with signed points, Riv. Mat. Sci. Econom. Social. 1 (1978) 81-88.

doi:10.1002/jgt.3190160104

[8] F. Harary, On the notion of balance of a signed graph, Michigan Math. J. 2 (195354) 143-146 and addendum preceding p. 1.

[9] C. Hoede, A characterization of consistent marked graphs, J. Graph Theory 16 (1992) 17-23. doi:10.1002/jgt.3190160104

[10] M. Joglekar, N. Shah and A.A. Diwan, Balanced group labeled graphs, Discrete Math. 312 (2012) 1542-1549. doi:10.1016/j.disc.2011.09.021

[11] S.B. Rao, Characterizations of harmonious marked graphs and consistent nets, J. Comb. Inf. Syst. Sci. 9 (1984) 97-112.

[12] F.S. Roberts and S. Xu, Characterizations of consistent marked graphs, Discrete Appl. Math. 127 (2003) 357-371. doi:10.1016/S0166-218X(02)00254-8

[13] T. Zaslavsky, Matrices in the theory of signed simple graphs, Advances in Discrete Mathematics and Applications: Mysore, 2008, B.D. Acharya, G.O.H. Katona, and J. Nesetril, Eds., Ramanujan Math. Soc., Mysore, India (2010) 207-229.

[14] T. Zaslavsky, Consistency in the naturally vertex-signed line graph of a signed graph, Bull. Malaysian Math. Sci. Soc., to appear.

Received 28 May 2014

Revised 14 December 2014

Accepted 22 January 2015 